\section{Is There a Correlation Between Infection Control Performance and Other Hospital Quality Measures?}

\author{
Lyndsay M. O'Hara, PhD, MPH; ${ }^{1}$ Daniel J. Morgan, MD, \\ MS; ${ }^{1}$ Lisa Pineles, MA; ${ }^{1}$ Shanshan Li, $\mathrm{PhD} ;{ }^{2}$ Carol Sulis, $\mathrm{MD} ;{ }^{3}$ \\ Jason Bowling, MD; $;^{4}$ Marci Drees, MD,MS; ${ }^{5,6}$ Jesse T. Jacob, \\ $\mathrm{MD} ;{ }^{7}$ Deverick J. Anderson, MD,MPH; ${ }^{8}$ David K. Warren, \\ $\mathrm{MD}, \mathrm{MPH} ;{ }^{9}$ Anthony D. Harris, MD, $\mathrm{MPH}^{1}$
}

Quality measures are increasingly reported by hospitals to the Centers for Medicare and Medicaid Services (CMS), yet there may be tradeoffs in performance between infection control (IC) and other quality measures. Hospitals that performed best on IC measures did not perform well on most CMS non-IC quality measures.

Infect Control Hosp Epidemiol 2017;38:736-739

In response to the movement toward patient-centered care, quality measures are increasingly being reported by hospitals to the Centers for Medicare and Medicaid Services (CMS). The CMS Hospital Compare website provides information regarding the quality of care at more than 4,000 hospitals and includes more than 120 measures. ${ }^{1}$ While tools such as Hospital Compare provide valuable information to healthcare consumers, providers, and administrators, there is concern that the metrics may not be consistent and that some may not provide an accurate depiction of quality in the institution. ${ }^{2}$ Furthermore, the CMS Value-Based Purchasing (VBP) program rewards hospitals with incentive payments for the quality of care they provide, ${ }^{3}$ yet recent studies found little evidence that public reporting of hospital quality measures ${ }^{4-6}$ and VBP rewards lead to improved patient outcomes. ${ }^{7}$ To our knowledge, no published studies have assessed infection control (IC) measures and their correlation with other outcomes. The primary aim of this study was, therefore, to ascertain whether hospitals that perform well in IC measures also perform well on CMS non-IC quality measures.

\section{METHODS}

This cross-sectional study included 20 acute-care hospitals from 16 states in the United States. A previous randomized controlled trial (RCT) was conducted in 20 intensive care units (ICUs) in 2012 to evaluate whether wearing gloves and gowns for all patient contact decreased acquisition of methicillinresistant Staphylococcus aureus (MRSA) or vancomycinresistant Enterococcus (VRE). ${ }^{8}$ Data regarding the following 6 IC measures were collected as part of this previous RCT for each ICU: (1) hand-hygiene compliance, (2) gown compliance, (3) glove compliance, (4) central line-associated bloodstream infection (CLABSI) rate, (5) catheter-associated urinary tract infection (CAUTI) rate, and (6) VRE or MRSA colonization acquisition rate.

The following 6 non-IC quality measures were selected by author consensus upon review of the CMS Hospital Compare program for the period available that was closest to 2012: (1) rate of hospital-wide readmission, (2) rate of serious complications, (3) nurse communication, (4) doctor communication, (5) hospital recommendation, and (6) timely venous thromboembolism prophylaxis.

\section{Infection Control Measures}

Hand-hygiene compliance was recorded as a proportion of observed hand-hygiene events divided by the number of times a healthcare worker exited a room. Compliance with glove and gown use was measured in the same way. CLABSI and CAUTI rates were calculated in accordance with National Healthcare Safety Network definitions as cases per 1,000 patient days. VRE or MRSA colonization acquisition data were obtained by active surveillance culturing on ICU admission and discharge. ${ }^{8}$

\section{CMS Non-Infection Control Quality Measures}

Rate of readmission included all unplanned hospital-wide 30-day readmissions. ${ }^{9}$ The serious complications variable was a composite of 11 measures from the Agency for Healthcare Research and Quality Patient Safety Indicators and was based on how often adult patients had certain serious but potentially preventable complications. ${ }^{9}$ Nurse and doctor communication and overall hospital recommendation were derived from linear mean scores from the Hospital Consumer Assessment of Healthcare Providers and Systems (HCAHPS) survey. ${ }^{9}$ The venous thromboembolism variable from the Joint Commission describes the percentage of surgery patients who received appropriate venous thromboembolism prophylaxis. ${ }^{9}$

Hospitals were assigned a rank for each variable. For VRE/ MRSA rate, CLABSI rate, CAUTI rate, rate of hospital-wide readmission, and rate of serious complications, lower rate values were assigned higher ranks (rank $1=$ best, rank $20=$ worst). Conversely, for hand-hygiene compliance, gown compliance, glove compliance, nurse communication, doctor communication, hospital recommendation, and timely venous thromboembolism prophylaxis, higher values were assigned higher ranks. To visually compare hospital performance, overall ranks for a composite of the IC outcomes and a composite of CMS non-IC quality measures were displayed graphically. To statistically compare the correlation between variables, Spearman's rank-order correlation coefficients $\left(r_{s}\right)$ were calculated for each variable pair. For this study, $r_{s}>0.80$ 
or $<-0.80$ was considered a strong correlation and $r_{s}$ between 0.50 and 0.80 or between -0.50 and -0.80 was considered a moderate correlation. ${ }^{10}$

\section{RES ULTS}

Hospitals that performed best on IC measures did not perform well on most CMS non-IC quality measures (Figure 1). For example, for overall IC scores versus the overall CMS non-IC outcome category, the hospitals that ranked $1^{\text {st }}, 2^{\text {nd }}$, and $3^{\text {rd }}$ on IC measures were ranked $10^{\text {th }}, 5^{\text {th }}$, and $8^{\text {th }}$, respectively, on CMS non-IC quality measures (Figure 1). Similarly, the top 3 hospitals for CMS non-IC quality measures were ranked $7^{\text {th }}$, $12^{\text {th }}$ and $13^{\text {th }}$ (tied for $2^{\text {nd }}$ rank), and $9^{\text {th }}$ for IC measures (Figure 1). Overall, there were only 8 significant correlations among 36 possible associations. We did not find any differences between the intervention group of the RCT compared to the control group.
Correlations Between Infection Control Measures and CMS Non-Infection Control Quality Measures

There were no strong correlations and only 2 moderate correlations between IC measures and CMS non-IC quality outcomes. Moderate correlations were observed between hand-hygiene compliance and nurse communication $\left(\mathrm{r}_{\mathrm{s}}=0.53 ; P=.016\right)$ and between VRE or MRSA rate and rate of readmission $\left(\mathrm{r}_{\mathrm{s}}=0.53 ; P=.02\right)$ (Table 1$)$.

\section{Correlations Among Infection Control Measures}

Among IC measures, there was 1 strong correlation and 2 moderate correlations. A strong correlation was observed between glove compliance and gown compliance $\left(r_{s}=0.92\right.$; $P \leq .0001)$, and moderate correlations were observed between hand-hygiene compliance and gown compliance $\left(r_{s}=0.68\right.$; $P=.001)$ as well as hand-hygiene compliance and glove compliance $\left(\mathrm{r}_{\mathrm{s}}=0.56 ; P \leq .01\right)($ Table 1$)$.

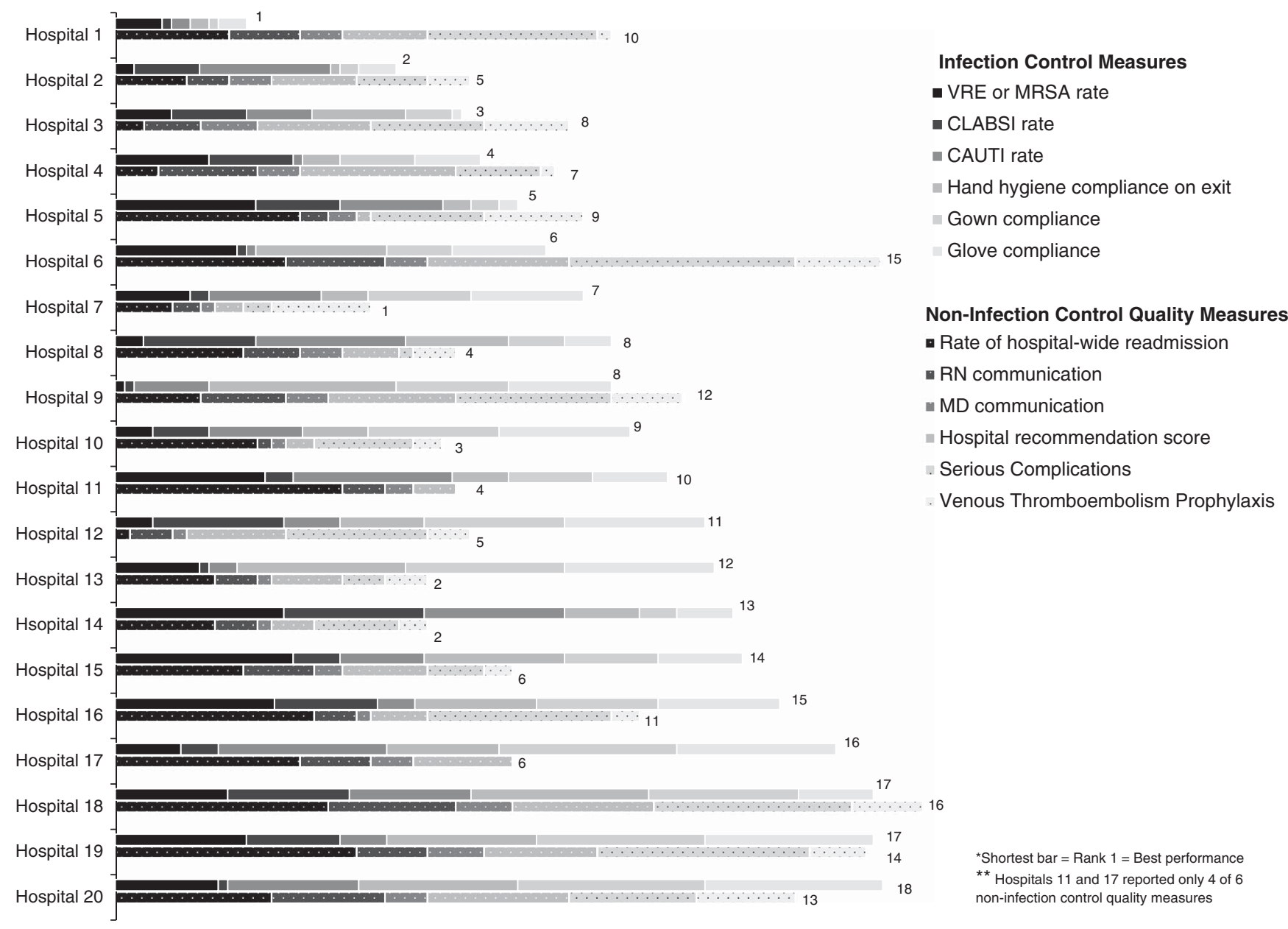

FIGURE 1. Hospital rankings by infection control measures and Centers for Medicare and Medicaid Services (CMS) non-infection control quality measures. 
TA BLE 1. Correlations Between Infection Control Measures and Centers for Medicare and Medicaid Services (CMS) Non-Infection Control Measures

\begin{tabular}{|c|c|c|c|c|c|c|c|c|c|c|c|c|}
\hline \multicolumn{13}{|c|}{ Spearman Correlation Coefficient ( $P$ Value) } \\
\hline \multicolumn{6}{|c|}{ Infection Control Measures } & \multicolumn{7}{|c|}{ CMS Non-Infection Control Quality Measures } \\
\hline & $\begin{array}{c}\text { Glove } \\
\text { Compliance }\end{array}$ & $\begin{array}{c}\text { Gown } \\
\text { Compliance }\end{array}$ & $\begin{array}{l}\mathrm{HH} \\
\text { Compliance } \\
\text { (on room } \\
\text { exit) }\end{array}$ & $\begin{array}{l}\text { CLABSI } \\
\text { Rate }^{\mathrm{a}}\end{array}$ & $\begin{array}{l}\text { CAUTI } \\
\text { Rate }^{\mathrm{a}}\end{array}$ & $\begin{array}{l}\text { VRE or } \\
\text { MRSA Rate }^{\mathrm{a}}\end{array}$ & $\begin{array}{l}\text { Hospital- } \\
\text { wide } \\
\text { Readmission }\end{array}$ & $\begin{array}{c}\text { Serious } \\
\text { Complications }\end{array}$ & $\frac{\mathrm{RN}}{\text { Communication }}$ & $\frac{\text { MD }}{\text { Communication }}$ & $\begin{array}{c}\text { Hospital } \\
\text { Recommend }\end{array}$ & $\begin{array}{c}\text { VTE } \\
\text { Prophylaxis }\end{array}$ \\
\hline \multicolumn{13}{|l|}{ Infection Control Measures } \\
\hline Glove compliance & 1.00 & & & & & & & & & & & \\
\hline Gown compliance & $.915(<.0001)$ & 1.00 & & & & & & & & & & \\
\hline HH compliance (on room exit) & $.557(.011)$ & $.681(.001)$ & 1.00 & & & & & & & & & \\
\hline CLABSI Rate $^{\mathrm{a}}$ & $-.221(.362)$ & $-.090(.705)$ & $-.130(.586)$ & 1.00 & & & & & & & & \\
\hline CAUTI Rate $^{\mathrm{a}}$ & $-.040(.867)$ & $.059(.803)$ & $-.083(.729)$ & $.174(.463)$ & 1.00 & & & & & & & \\
\hline VRE or MRSA Rate ${ }^{a}$ & $.052(0.828)$ & $0.055(.818)$ & $.138(.561)$ & $.182(.443)$ & $-.023(.922)$ & 1.00 & & & & & & \\
\hline \multicolumn{13}{|c|}{ CMS Non-Infection Control Quality Measures } \\
\hline Hospital-wide Readmission & $.258(.272)$ & $.318(.171)$ & $.297(.203)$ & $.017(.943)$ & $.231(.328)$ & $.531(.016)$ & 1.00 & & & & & \\
\hline Serious complications & $.183(.468)$ & $.230(.358)$ & $.306(.217)$ & $.007(.977)$ & $-.437(.070)$ & $.207(.411)$ & $.493(.038)$ & 1.00 & & & & \\
\hline RN communication & $.105(.661)$ & $.232(.323)$ & $.529(.016)$ & $-.188(.428)$ & $-.187(.429)$ & $.043(.856)$ & $.154(.517)$ & $.456(.038)$ & 1.00 & & & \\
\hline MD communication & $-.215(.272)$ & $-.026(.913)$ & $.230(.329)$ & $-.039(.869)$ & $-.018(.429)$ & $-.188(.427)$ & $.178(.453)$ & $.401(.099)$ & $.730(.003)$ & 1.00 & & \\
\hline Hospital Recommend & $.181(.445)$ & $.292(.212)$ & $.469(.037)$ & $-.126(.598)$ & $-.351(.129)$ & $-.126(.596)$ & $-.084(.726)$ & $.487(.041)$ & $.898(<.0001)$ & $.696(.0006)$ & 1.00 & \\
\hline VTE prophylaxis & $.099(.695)$ & $.200(.424)$ & $.284(.254)$ & $-.211(.400)$ & $.280(.260)$ & $-.019(.941)$ & $.138(.584)$ & $.139(.583)$ & $.067(.792)$ & $.258(.301)$ & $.093(.715)$ & 1.00 \\
\hline
\end{tabular}

NOTE. HH, hand hygiene; CLABSI, central line-associated bloodstream infection; CAUTI, catheter-associated urinary tract infection; VRE, vancomycin-resistant Enterococcus; MRSA, methicillin-resistant Staphylococcus aureus; RN, nurse; MD, doctor; VTE, venous thromboembolism.

Bold values indicate correlations where $P<.05$

${ }^{\mathrm{a}}$ Cases per 1,000 patient days. 


\section{Correlations Between CMS Non-Infection Control Quality Measures}

Among CMS non-IC quality measures, there was 1 strong correlation and 2 moderate correlations. A strong correlation was observed between overall hospital recommendation and nurse communication $\left(\mathrm{r}_{\mathrm{s}}=0.90 ; \quad P \leq .0001\right)$. Moderate correlations were observed between overall hospital recommendation and doctor communication $\left(\mathrm{r}_{\mathrm{s}}=0.70 ; P=.0006\right)$ as well as nurse communication and doctor communication $\left(\mathrm{r}_{\mathrm{s}}=0.73 ; P=.003\right)($ Table 1$)$.

\section{DISCUSSION}

The lack of correlations in our results reinforces the hypothesis that there are hospital-level differences in IC measures and other non-IC quality measures. These findings also suggest that some of the metrics currently reported by CMS may not appropriately depict hospital quality. This study provides insights into the potential tradeoffs between IC measures and other quality measures. The reasons for the lack of observed correlations are likely multifactorial and may include the following: (1) Hospitals have insufficient resources to tackle multiple hospital quality measures and thus focus only on a few. (2) Some hospital quality measures should be re-evaluated regarding their causal relationship to quality. (3) Better risk adjustment must be done for quality measures to ensure that hospitals with complex patient populations are not inappropriately penalized for metrics such as infection rates.

This study has several limitations. First, it has cross-sectional design, and measurement periods do not always overlap. Furthermore, the IC measures were based solely on a single ICU, whereas all non-IC measures from Hospital Compare were based on whole-hospital performance. In addition, most hospitals included were academic teaching centers; thus, these results may not be generalizable to all hospitals.

Public reporting is an important part of hospital IC and quality; however, presenting too many metrics can be confusing to healthcare consumers and may not provide an appropriate picture of hospital performance. In addition, the potential pros and cons of value-based purchasing by CMS has been heatedly debated. More work is needed to analyze the outcomes and method of data presentation currently utilized by the CMS. To fully optimize the VBP model, national efforts that focus on patient experiences, such as the CMS VBP program, must validate that they are rewarding quality instead of inadvertently punishing high-performing hospitals that may not perform well on certain measures.

\section{ACKNOWLEDGMENTS}

Financial support: L.M.O. is the recipient of a Banting Postdoctoral Fellowship administered by the Government of Canada. A.D.H. received grant support from the National Institutes of Health (K24) and the Agency for Healthcare Research and Quality (grant nos. 1R18HS024045-01 and HHSA290200600015).
This study was not funded by industry and no manufacturer played a role in the gathering or preparation of data or in the writing of the manuscript.

Potential conflicts of interest: All authors report no conflicts of interest relevant to this article.

Affiliations: 1. Department of Epidemiology and Public Health, University of Maryland School of Medicine, Baltimore, Maryland; 2. Department of Biostatistics, Indiana University R.M. Fairbanks School of Public Health, Indianapolis, Indiana; 3. Boston Medical Center, Boston, Massachusetts; 4. University of Texas Health Science Center, San Antonio, Texas; 5. Christiana Care Health System, Wilmington, Delaware; 6. Sidney Kimmel Medical College, Thomas Jefferson University, Philadelphia, Pennsylvania; 7. Division of Infectious Diseases, Department of Medicine, Emory University School of Medicine, Atlanta, Georgia; 8. Duke Center for Antimicrobial Stewardship and Infection Prevention, Duke University, Durham, North Carolina; 9. Department of Medicine, Washington University, St. Louis, Missouri.

Address correspondence to Dr. Anthony Harris, Epidemiology and Public Health, University of Maryland School of Medicine, 685 W. Baltimore Street, MSTF 330, Baltimore, MD 21201 (aharris@epi.umaryland.edu).

Received August 31, 2016; accepted: January 24, 2017; electronically published April 5, 2017

(C) 2017 by The Society for Healthcare Epidemiology of America. All rights reserved. 0899-823X/2017/3806-0017. DOI: 10.1017/ice.2017.27

\section{REFERENCES}

1. U.S. Government Site for Medicare Hospital Compare website. https://www.medicare.gov/hospitalcompare/search.html. Accessed June 10, 2016.

2. Tsai TC, Orav EJ, Jha AK. Patient satisfaction and quality of surgical care in US hospitals. Ann Surg 2015;26:2-8.

3. Hospital value-based purchasing. Centers for Medicare and Medicaid Services website. https://www.cms.gov/Medicare/ Quality-Initiatives-Patient-Assessment-Instruments/hospitalvalue-based-purchasing/. Published 2016. Accessed June 27, 2016.

4. Fung CH, Lim YW, Mattke S, Damberg C, Shekelle PG. Systematic review: the evidence that publishing patient care performance data improves quality of care. Ann Internal Med 2008;148:111-123.

5. Hibbard JH. What can we say about the impact of public reporting? Inconsistent execution yields variable results. Ann Internal Med 2008;148:160-161.

6. Bangalore S, Guo Y, Xu J, Blecker S, Gupta N, Feit F, Hochman JS. Rates of invasive management of cardiogenic shock in New York before and after exclusion from public reporting. JAMA Cardiol 2016;1:640-647.

7. Figueroa JF, Tsugawa Y, Zheng J, Orav EJ, Jha AK. Association between the value-based purchasing pay for performance program and patient mortality in US hospitals: observational study. BMJ 2016;353:i2214.

8. Harris AD, Pineles L, Belton B, et al. Universal glove and gown use and acquisition of antibiotic-resistant bacteria in the ICU: a randomized trial. JAMA 2013;310:1571-1580.

9. About the data: measures and current data collection periods. U.S. Government Site for Medicare Hospital Compare website. https:// www.medicare.gov/hospitalcompare/Data/Data-Updated.html\#. Published 2016. Accessed June 1, 2016.

10. Bleavins MR, Carini C, Jurima-Romet M, Rahbari R. editors Biomarkers in Drug Development: A Handbook of Practice, Application, and Strategy. Hoboken, NJ: Wiley; 2011. 